\title{
DEVELOPMENT OF THE TEST SYSTEM FOR MEASURING THE IMPACT ENERGY OF A HYDRAULIC BREAKER
}

\author{
Jong-Won PARK*, Hyoung-Eui KIM* \\ * Hydraulic and Pneumatic Group, Department of Advanced Industrial Technology \\ Korea Institute of Machinery and Materials \\ 171 Jang Dong, Yu Sung Gu, Taejun, 305-600, South Korea \\ (E-mail: jwpark@kimm.re.kr)
}

\begin{abstract}
A hydraulic breaker for construction machinery generally used for the destroying and disassembling of buildings, crashing road pavement, breaking rocks at quarry and so on. So the measurement of the impact energy of a hydraulic breaker is very important thing to prove its capability to manufacturers and customers. In this study, the test system for measuring the impact energy of a hydraulic breaker was designed and constructed. The test system was consisted with hydraulic cylinders for mounting a breaker, impact absorbing base and frames, pressure and flow sensors, high speed and accurate data acquisition system, diesel engine driven hydraulic power pack and etc. The test process of the developed system was carried by measuring guide for tool energy rating for hydraulic breakers which was developed by the CIMA (Construction Industry Manufacturers Association) USA. The developed test system can be applied to measure the impact energy for various kind of hydraulic breakers.
\end{abstract}

\section{KEY WORDS}

Hydraulic breaker, Measuring impact energy, Test system, Strain gauge equipment, Tool impact energy calculation

\section{NOMENCLATURE}

$A_{t}$ : Cross-sectional area of the test tool at strain gage assembly location

$C F$ : Correction factor from static calibration

$E_{i}:$ A single blow impact energy of the tool

$E_{t}$ : Young's modulus of the test tool material

$f_{i}$ : Blow frequency

$H$ : Distance between the strain gage assembly and the tip of the test tool

$k$ : Gage factor (must be the same for all active gages)

$K_{a}$ : Amplification factor of the amplifier $\left(K_{a}=1\right.$, if amplifier not used)

$L_{p}$ : Length of the piston

$\rho_{t}$ : Mass density of the test tool material

$P_{\text {in }}$ : Total hydraulic input power
$P_{s}:$ Hydraulic supply line pressure

$P_{r}:$ Hydraulic return line pressure

$Q_{i}$ : Breaker inlet oil flow

$T$ : Operating oil temperature

$t:$ Time

$t_{1}:$ Starting time of integration

$t_{n}$ : Ending time of integration

$\Delta t_{i}:$ Integration interval, $\Delta t_{i}=\left(t_{n i}-t_{l i}\right)$

$U$ : Bridge output voltage

$U_{B}$ : Bridge supply voltage

$\varepsilon_{i}:$ Measured strain

$v$ : Oil kinematic viscosity

\section{INTRODUCTION}

A hydraulic breaker for construction machinery 
generally used for the destroying and disassembling of buildings, crashing road pavement, breaking rocks at quarry and so on. A hydraulic breaker is composed of cylinder, piston, valve, back head, chisel and power cell and these components are fixed by bracket. The section view of the general structure of a hydraulic breaker is shown in Figure 1.

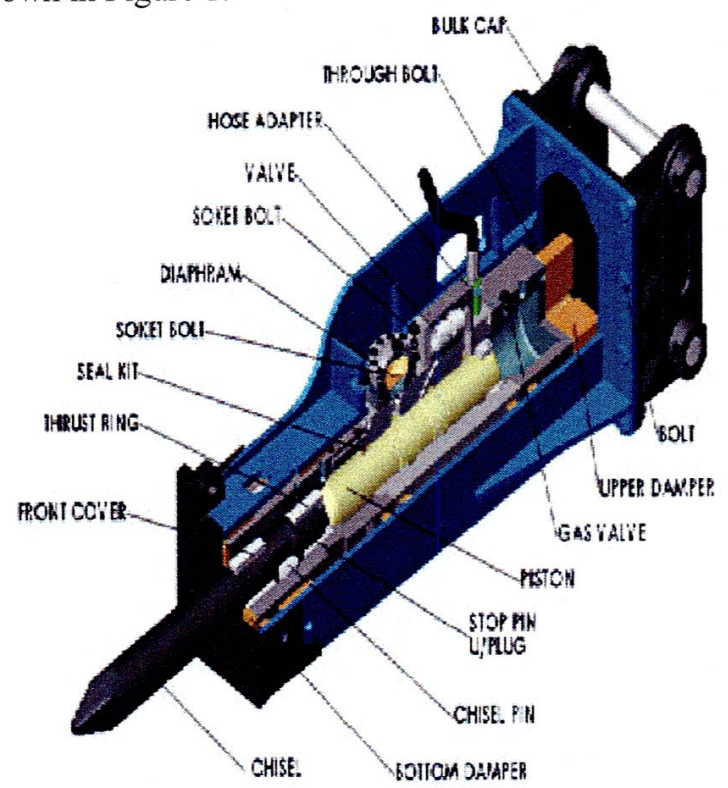

Figure 1 General structure of a hydraulic breaker

A hydraulic breaker blows an object using kinetic energy that is transferred by impacting chisel with reciprocating piston via hydraulic power from an excavator. So the measurement of the impact energy of a hydraulic breaker is very important thing to prove its capability to manufacturers and customers. But a hydraulic breaker is operated by very large kinetic energy, so that the accurate measurement of impact force has been considered too difficult problem.

In this study, the test system for measuring the impact energy of a hydraulic breaker was designed and constructed. The test system was consisted with hydraulic cylinders for mounting a breaker, impact absorbing base and frames, pressure and flow sensors, high speed and accurate data acquisition system, diesel engine driven hydraulic power pack and etc. In addition the process of measuring impact energy and the consideration of the test result was discussed.[1][2][3]

\section{DEVELOPMENT OF TEST SYSTEM}

The test system, shown in Figure 2, was developed to evaluate the impact energy of hydraulic breakers. As shown in Figure 2, the developed test system holds a breaker by 4 hydraulic cylinders and can evaluate the impact energy for various sizes of breakers by adjusting the stroke of 4 hydraulic cylinders. In addition to, the developed system has another hydraulic cylinder on the upper part of it to regulate blowing angle in testing.

Target base was constructed with accumulating vibration isolating materials like as sands, broken stones, trees and rubbers after $5 \mathrm{~m}$ depth foundation work to absorb shock and vibration at blowing. The schematic diagram of the target base is shown in Figure 3.

Hydraulic power for operating breaker is supplied by tandem pump that has maximum rated supply flow rate 580LPM and maximum rated supply pressure 450 bar. The tandem pump is driven by diesel engine to realize similar operating condition of excavators. The tandem pump and diesel engine that was equipped in the developed test system are shown in Figure 4.

Data acquisition equipment has above $125 \mathrm{kHz}$ cutoff frequency and $4 \mathrm{MHz}$ sampling frequency to detect shock wave accurately. The data acquisition equipment is controlled by real time operating system and software was developed to carry test process automatically. [5]

Pressure, turbine type flow and temperature sensors are used for monitoring the status of test system and breaker and measuring parameters to evaluate impact energy. Data acquisition equipments are shown in Figure 5 and the schematic diagram of the hydraulic circuit of the test system is shown in Figure 6.

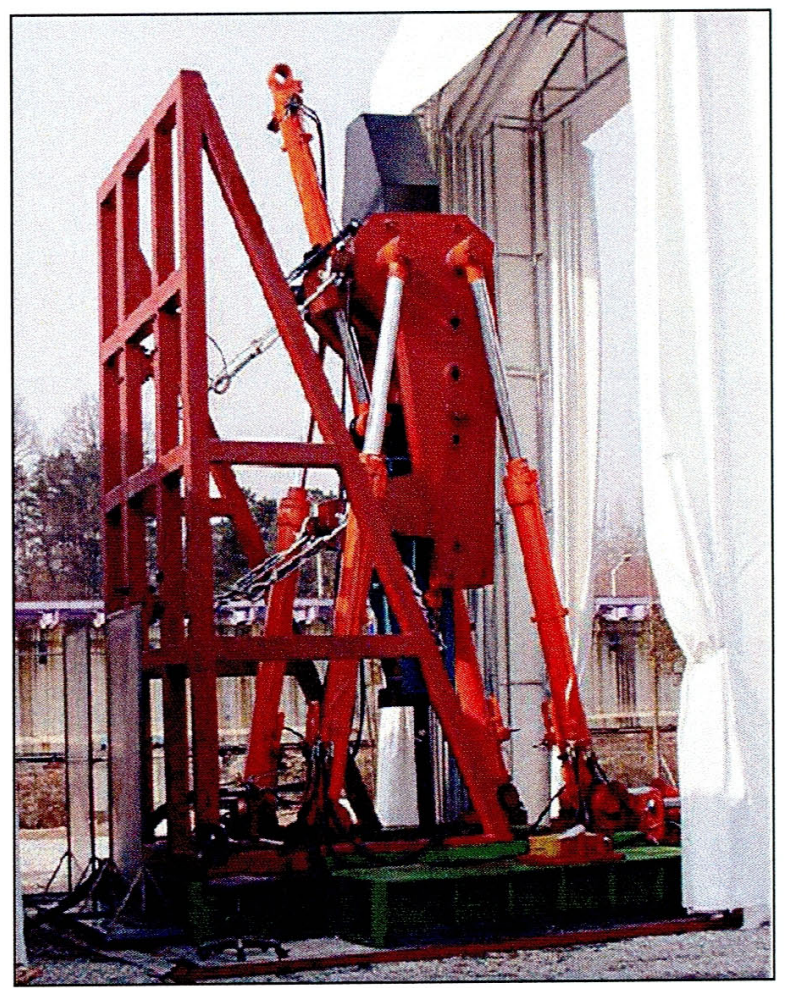

Figure 2 Mechanism of test system 


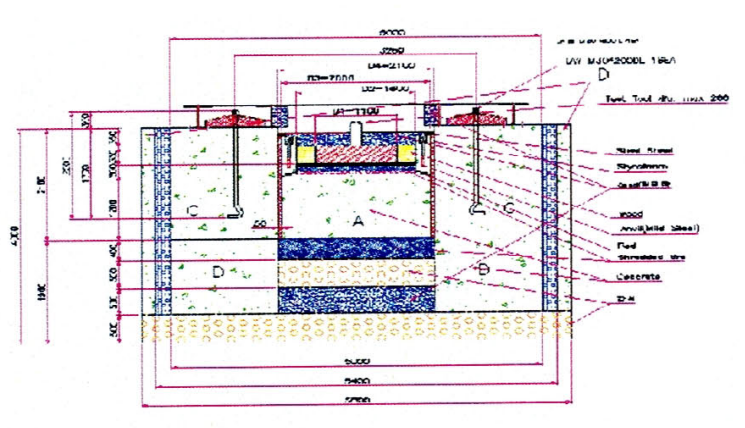

Figure 3 Target base of test system
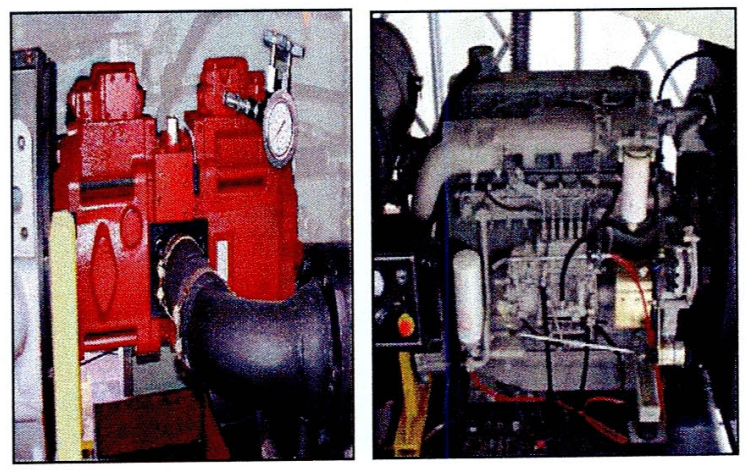

Figure 4 Hydraulic power unit of test system

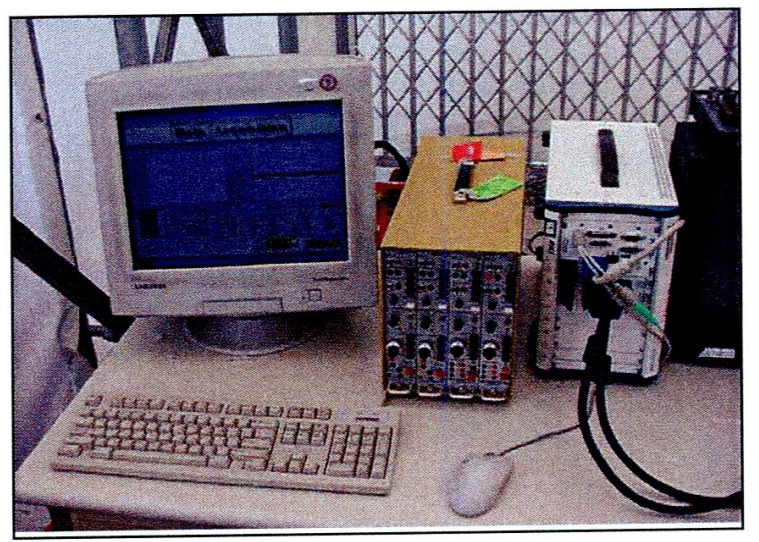

Figure 5 Data acquisition equipment of test system

\section{IMPACT ENERGY EVALUATION}

The developed test system evaluates the impact energy of hydraulic breakers, after measuring the strain of chisel with strain gauge.[4] Strain gauge attached test tool is shown in Figure 7.[6][7]

To evaluate the impact energy of a hydraulic breaker, we have to proceed as following process.

Single blow impact energy can be calculated from the measured strain using the equation (1) and this can be derived by elastic strain energy method for an impact loading.

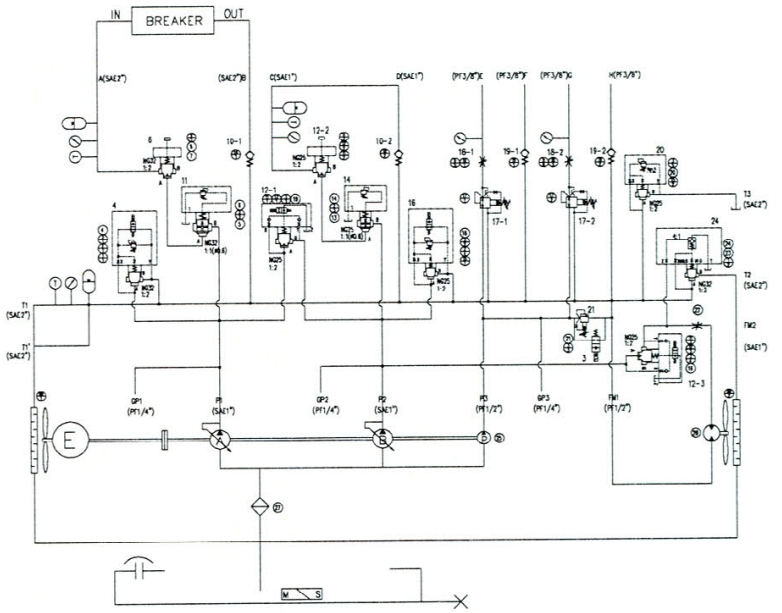

Figure 6 Schematic diagram of test system

$$
E_{i}=\frac{C F^{2}}{A_{t} \cdot \sqrt{E_{t} \cdot \rho_{t}}} \int_{t_{1}}^{t_{i}} \varepsilon_{i}^{2} \cdot d t
$$

\section{Where, $\varepsilon_{\mathrm{i}}=4 \mathrm{U} /\left(2.6 \mathrm{k} \mathrm{U}_{\mathrm{B}} \mathrm{K}_{\mathrm{a}}\right)$}

The integration interval $t_{1}$ through $t_{n}$ must be defined for each measurement to identify and isolate the effect of the hydraulic breaker static preload on the test tool strain and to prevent accumulation of stress from the next pulse or reflected stress wave.

The 25 energy measurements must be recorded in a steady state operation with test breaker's specifications.

The 25 measurements can be divided into sets of 5 consecutive blows minimum. Each measured energy value shall be within $\pm 10 \%$ of the average value calculated in final tool impact energy. Final tool impact energy can be calculated by equation (2). Energy values out of this range must be disregarded and measurement shall be taken again. Total output efficiency can be calculated by equation (3).

$$
\begin{aligned}
& E=\frac{1}{25} \sum_{i=1}^{25} E_{i} \\
& \eta_{t o t}=\frac{E \cdot f}{\left(P_{s}-P_{r}\right) \cdot Q}
\end{aligned}
$$

The complete measuring equipment and installation must be calibrated by comparing the strain $\varepsilon_{\text {meus }}$ calculated from the measuring equipment output voltage with the calibrated force $F_{\text {cal }}$ applied on the test tool and measured by a calibrated load cell. Calibration will be carried out by compressing the test tool (without breaker) against a force transducer (load cell) for example in a hydraulic press. Correction factor $C F$ can be calculated by equation (4). 


$$
\mathrm{CF}=\frac{\mathbf{F}_{\text {cal }}}{\varepsilon_{\text {meas }}}
$$

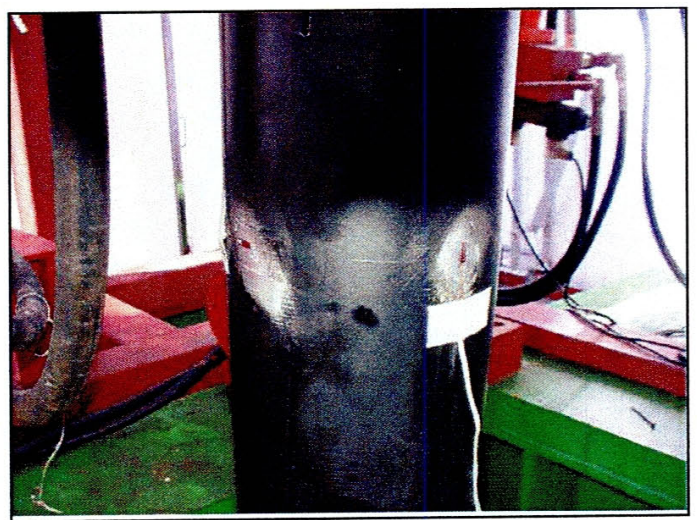

Figure 7 Strain gauge attached chisel

\section{TEST RESULT}

\section{Detection of the Shock Wave}

The detected shock wave of impact energy strain is shown in Figure 8 and the test result of blow frequency is shown in Figure 9. The integration limits $t_{1}$ through $t_{n}$ must be defined for each pulse to identity and isolate the effect of the static preload of the breaker on the test tool strain and to prevent accumulation of the strain from the next pulse. Before impact, the test tool will begin to unload due to recoil of the hydraulic breaker body. Time $t_{l}$ must be measured in this region of the curve. The baseline of integration will be taken at the point $t_{1}$. The last time recorded, $t_{n}$, for each pulse, must be separate and distinct from the next pulse or reflected stress wave. Time $t_{n}$ should be measured at the point where the pulse curve becomes negative in relationship with the baseline. Integration will stop when the curve reaches the same strain level that was found at time $t_{l}$. However, the maximum integration interval $t_{n}-t_{l}$ must be less than $2 H / c_{t}$ with, $c_{t}=\sqrt{E_{t} / P_{t}}$. Usually the distance $H=$ $1.2 L_{p}$ is sufficient, if not, the stress wave may combine with the reflected wave causing incorrect instrumentation readings and energy results. This can occur especially, when the piston cross-sectional area $A_{p}$ is greater than the tool cross-sectional area $A_{t}$. In this case the test tool is too short and distance $H$ should be at least $2 L_{p}$. [4]

\section{Total uncertainty of the test result}

From equation (1), the uncertainty of the tool impact energy measurement can be calculated by equation (5) and the total uncertainty with $\pm 2 \sigma$ value, $95.7 \%$ probability in normal distribution can be calculated by equation (6).

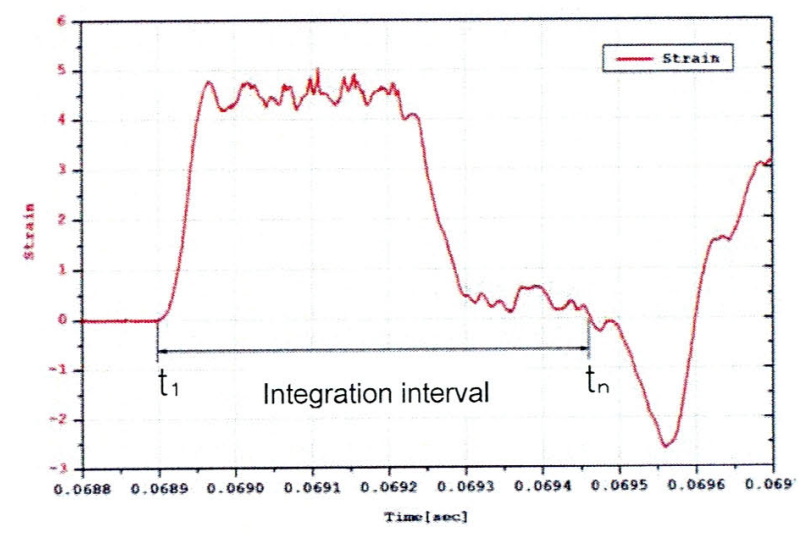

Figure 8 Shock wave of impact energy strain

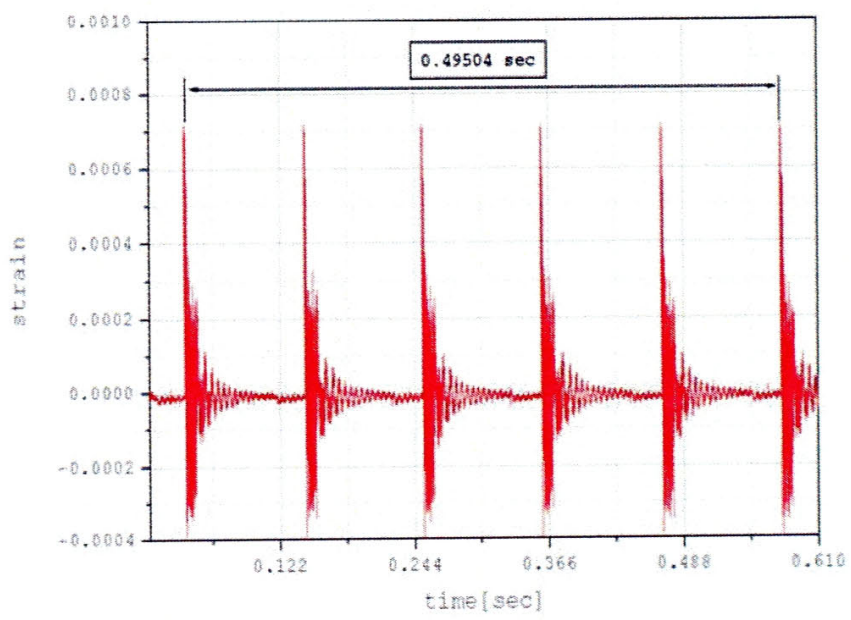

Figure 9 Test result of blow frequency

$\Delta E=2 \cdot|\Delta C F|+\left|-\Delta A_{t}\right|+\frac{1}{2} \cdot\left|-\Delta E_{t}\right|+\frac{1}{2} \cdot\left|-\Delta \rho_{t}\right|+\mid \Delta$ Integ $\mid$

$U_{t o t, \pm 2 \sigma}= \pm \frac{2}{3} \cdot \sqrt{(\Delta E)^{2}+\left(\Delta p_{S}\right)^{2}+\left(\Delta p_{a}\right)^{2}+(\Delta \varepsilon)^{2}}$

The maximum allowed uncertainties of the measured parameters in the equation (5) and (6) are as follows.

$$
\begin{aligned}
& \Delta P_{S}= \pm 1 \%, \Delta P_{a}= \pm 2 \%, \Delta \varepsilon= \pm 1 \%, \Delta C F= \pm 1.5 \% \\
& \Delta A_{t}= \pm 0.2 \%, \Delta E_{t}= \pm 3 \%, \Delta \rho_{t}= \pm 0.4 \%, \Delta \text { Integ }= \pm 0.2 \%
\end{aligned}
$$

By substituting the maximum allowed uncertainties of 
the measured parameters to the equation (6), $U_{t o t, 2 \sigma}$ can be evaluated as follows.[4]

$$
U_{t o t, \pm 2 \sigma}= \pm 3.8 \%
$$

In this study, the total uncertainty was calculated to $3.74 \%$. So the developed test system could satisfy the allowed uncertainties.

\section{CONCLUDING REMARKS}

In this study, the test system for measuring the impact energy of a hydraulic breaker was designed and constructed. The test system was consisted with hydraulic cylinders for mounting a breaker, impact absorbing base and frames, pressure and flow sensors, high speed and accurate data acquisition system, diesel engine driven hydraulic power pack and etc. The test process of the developed system was carried by measuring guide for tool energy rating for hydraulic breakers which was developed by the CIMA (Construction Industry Manufacturers Association) USA. The developed test system can be applied to measure the impact energy for various kind of hydraulic breakers and was certified to test cite for breakers by AEM(The Association of Equipment Manufacturers).

\section{REFERENCES}

1. Geun-Ho Lee, Young-Bum Lee, Ki-Young Lee, Development the Test System of Impact Energy Using the Pressure Variation in Closed Vessel for Hydraulic Breaker, Transactions of Korea Institute of Machinery and Materials, 2002, Vol. 32, pp.45-53.(in Korean)

2. Kwihyun Choi, Changseop Song, A Study on the Performance Improvement of a Hydraulic Breaker, Transactions of Korean Society of Precision Engineering, 1998, pp.1028-1031.(in Korean)

3. Wonjune Sung, Taebong Noh, Changseop Song, A Study on the Reducing the Return Line Pressure Fluctuation of the Hydraulic Breaker System, Journal of Korean Society of Precision Engineering, 2002, Vol. 194.(in Korean)

4. CIMA (Construction Industry Manufacturers Association), Measuring Guide for Tool Energy Rating for Hydraulic Breakers, Milwaukee, USA, 1996

5. IEEE 1057, Digitizing Waveform Recorders

6. ASTM E251, Test Method for Performance Characteristics of Metallic Bonded Resistance Strain-gages

7. NAS 942, Strain-gages Bonded Resistance

8. John Watton, Fluid Power Systems, Prentice Hill, 1989

9. Ferdinand P. Beer, E. Russell Johnston Jr, Vector
Mechanics for Engineers $2^{\text {nd }}$ S.I. Metric Edition, McGraw-Hill, 1990 